\title{
Construção de uma Escala para Avaliar Atitudes de Estudantes de Medicina
}

\author{
The Construction of a Scale to Measure \\ Medical Students' Attitudes
}

Silvana Maria de Miranda ${ }^{I}$; Maria Marlene de Souza Pires ${ }^{I I}$; Silvia Modesto NassarIII; Carlos Alberto Justo da SilvaIII

\author{
PALAVRAS-CHAVE: \\ - Educação Médica. \\ - Atitude. \\ - Psicometria. \\ - Currículo. \\ - Estudantes de Medicina.
}

\section{KEYWORDS:}

- Education, Medical.

- Attitude.

- Psychometrics.

- Curriculum.

- Students, Medical.

Recebido em: 11/11/2007

Reencaminhado em: 24/01/2008

Reencaminhado em: 18/04/2008

Aprovado em: 07/05/2008

REVISTA BRASILEIRA DE EDUCAÇ̃̃o MÉDICA $104 \frac{133(1 \text { Supl. 1): } 104110 ; 2009}{200}$
${ }^{I}$ Universidade do Extremo Sul Catarinense, Santa Catarina, Brasil.

"Universidade Federal de Santa Catarina, Santa Catarina, Brasil; Hospital Infantil Joana de Gusmão, Santa Catarina, Basil.

III Universidade Federal de Santa Catarina, Santa Catariana, Brasil. 


\section{INTRODUÇÃO}

A atitude, considerada parte importante das habilidades afetivas, pode ser ensinada e apreendida. Do mesmo modo, pode ser influenciada por componentes cognitivos, motivacionais e emocionais. Seu poder presumido de influenciar a resposta do sujeito a um objeto social tem determinado o interesse de diversos pesquisadores em busca de técnicas de mensuração e promoção de mudanças atitudinais. A possibilidade de a atitude ser mensurada foi demonstrada por Thurstone (1928) e Likert (1932), entre outros estudiosos ${ }^{1-7}$.

A educação médica, a exemplo de outras áreas de conhecimento, abrange um conjunto de habilidades e atitudes que objetivam a preparação integral do estudante para a prática profissional. No caso do médico, essa preparação, em seu sentido mais amplo, envolve uma boa habilidade para tratar o paciente e, por extensão, a própria sociedade.

Assim, a atitude positiva esperada do estudante/profissional na relação médico-paciente-equipe de saúde é a adoção de padrões socialmente aceitos e inerentes ao conhecimento e/ou exercício da profissão. Como é sabido, o ambiente de aprendizagem pode influenciar de modo significativo a formação e a manutenção de atitudes decorrentes da motivação pessoal, de conflitos de valores, da condição técnica e de condições socioeconômicas ${ }^{1-3,7}$.

O desenvolvimento de atitudes positivas quanto a aspectos comuns a todos os estudantes e a influência psicológica exercida pela escola médica motivaram a busca de técnicas de mensuração e intervenção para diagnóstico e planejamento estratégico curricular, com a finalidade de alcançar as competências estabelecidas para o exercício profissional. As pesquisas para acompanhamento de valores de atitudes de estudantes de Medicina se iniciaram com conceitos como humanitarismo e cinismo. Nos últimos anos, foram desenvolvidos vários métodos para mensurar as características que os estudantes adquirem durante o período de graduação e que podem predizer a boa prática médica. Muitas escalas foram construídas, considerando objetivos educacionais da instituição, de especialidades médicas, de segmentos sociais ou em declarações de reconhecimento da aquisição de determinada habilidade ${ }^{1-4,7-17}$.

Miller ${ }^{1}$ considerou que o importante para esse tipo de estudo seria a filosofia geral da profissão médica, com atitudes construtivas e aprovadas, socialmente e pelos membros da profissão, não considerando vital que o estudante tenha ou não atitudes favoráveis a segmentos sociais ou a práticas isoladas.

Nesse contexto, a presente pesquisa teve como propósito construir e validar uma escala de atitude, do tipo Likert, adaptada à realidade sociocultural brasileira, com a contextualização da escola de Medicina no cenário nacional, adequada ao idioma pátrio e com a finalidade de permitir o diagnóstico e o acompanhamento da atitude de estudantes durante a graduação.

A atitude foi conceituada de maneira multidimensional, abrangendo diversos aspectos atitudinais representados por uma síntese hipotética de um conjunto de comportamentos reais ou de realidades mentais, considerando o delineamento do perfil profissional do médico preconizado pelas Diretrizes Curriculares Nacionais do Curso de Graduação em Medicina (Resolução CNE/CES nº 4, de 7 de novembro de 2001).

Tal delineamento estabeleceu como objetivo a formação de um médico generalista, humanista, crítico e reflexivo, com capacidade de atuar no processo de saúde-doença em seus diferentes níveis de atenção, pautado em princípios éticos e atitudes voltadas para a saúde, a cidadania, a comunidade, a aprendizagem, a comunicação, a atuação em equipe e o conhecimento e a compreensão do ser humano em sua totalidade.

As atitudes abordadas neste estudo foram relacionadas aos aspectos sociais da profissão médica, a saber: importância da ambiência física e social, reconhecimento e compreensão de crenças, ética (profissional e acadêmica) e conhecimento. Assim, busca-se estudar o direcionamento atitudinal aos aspectos levantados, independentemente do desenvolvimento e reconhecimento de capacidades cognitivas e de habilidades por parte do estudante.

O estudo traz ainda para reflexão o compromisso das escolas médicas na promoção de atitudes apropriadas ao exercício da medicina e necessárias à mediação entre competência clínica (habilidade e conhecimento) e prática clínica (habilidade-conhecimento-ação), sem o que não é possível oportunizar a formação integral do graduando.

\section{MÉTODO}

O presente estudo, desenvolvido segundo critérios metodológicos de coorte, observacional, quantitativo, exploratório e descritivo, atendeu às diretrizes e normas regulamentadoras de pesquisas que envolvem seres humanos. Foi aprovado pelo Comitê de Ética da Universidade do Extremo Sul Catarinense (Unesc) e identificado como Projeto 115/204.

A pesquisa foi realizada em um curso de graduação em Medicina, na cidade de Criciúma, em Santa Catarina, no ano de 2006.

O currículo do curso integra conhecimentos básicos e clínicos, mantendo um entrelaçamento de aprendizagem de órgãos e sistemas do ser humano, numa visão bioneuropsicosso- 
cial na qual o estudante constrói seu conhecimento, suportado ininterruptamente por disciplinas como Bioética, Psicologia Médica, Qualidade de Vida, Epidemiologia, além de laboratórios específicos e de habilidades médicas e laboratórios de informática. Há ainda ambulatórios de interação comunitária e ambulatórios clínicos, que permitem inserir o estudante na comunidade desde a primeira fase do curso.

A estrutura curricular integraliza inter/intra semestres letivos, implementa o processo de ensino-aprendizagem com base na solução de problemas e permite o melhoramento contínuo dos sistemas de avaliação do processo de ensino-aprendizagem e do próprio curso de graduação em Medicina.

A população do estudo foi constituída de 233 estudantes das oito primeiras fases, regularmente matriculados no primeiro semestre letivo de 2005.

A amostra foi composta por 202 estudantes que ingressaram no curso mediante exame vestibular e também participaram das duas aplicações da escala.

Na construção da escala de atitudes, o modelo adotado foi do tipo Likert, que objetiva verificar o nível de concordância do indivíduo com uma proposição que expressa algo favorável ou desfavorável em relação a um objeto psicológico. Espera-se que os indivíduos que apresentem atitudes favoráveis a determinado tema possivelmente concordem com itens que expressem algo positivo sobre a questão, e aqueles com atitudes negativas concordem com itens que expressem aspectos desfavoráveis ao tema e discordem daqueles que salientem pontos positivos ${ }^{1-7,18}$.

A avaliação enfocou cinco dimensões: social, ambiência, conhecimento, crenças e ética. A ética permeia as quatro primeiras dimensões e foi considerada a quinta dimensão especificamente quando aborda a percepção do comportamento ético do indivíduo enquanto acadêmico e profissional de Medicina.

Foram construídos 182 itens da escala para representar os construtos de interesse, expressando um tipo de comportamento. Os itens construídos se basearam inicialmente em referencial teórico específico da área da saúde. A redação procurou ser objetiva, simples, clara, sem ambigüidades e sem uso de expressões extremadas, com frases condizentes com o atributo. Com a intenção de discriminar e diferenciar os sujeitos, assim como evitar a repetição de respostas, formulou-se metade dos itens em termos favoráveis e metade em termos desfavoráveis.

O procedimento de validação aparente e de conteúdo foi realizado por um grupo de seis juízes, docentes das áreas de Medicina e Educação, todos com experiência pessoal e profissional importante no assunto investigado. Aos juízes foi solici- tado que opinassem sobre adequação semântica, facilidade de compreensão, adequação da representação comportamental e vinculação dos itens propostos em relação às respectivas dimensões para validação interna de conteúdos e construtos.

Dessa avaliação foram excluídos 80 itens, e a escala, em sua versão final, contou com 102 itens, assim distribuídos:

Dimensão social: 24 itens, sendo 12 representativos de atitudes positivas e 12 de atitudes negativas. Os aspectos analisados estavam relacionados à responsabilidade social (garantia de atendimento em todos os níveis de complexidade, interação com a comunidade, utilização racional de recursos diagnósticos e terapêuticos, e prevenção de doenças), à relação médico-paciente (percepção da relação médico-paciente em seus aspectos sociais, psicológicos, emocionais e autonomia) e à comunicação (aprendizagem da habilidade de comunicação e comunicação enquanto profissional médico);

Dimensão ambiência: 16 itens, sendo 8 representativos de atitudes positivas e 8 de atitudes negativas, todos relacionados à ecomedicina, ao trabalho em equipe e ao ambiente de aprendizagem e de trabalho;

Dimensão crenças: 16 itens, sendo 6 representativos de atitudes positivas e 10 de atitudes negativas, referentes às crenças derivadas do imaginário da profissão médica presentes na sociedade e à possível internalização de outras a partir de sistemas referenciais fornecidos pela ambiência do curso, além do respeito e compreensão das crenças do paciente e equipe de saúde;

Dimensão conhecimento: 30 itens, sendo 15 representativos de atitudes positivas e 15 de atitudes negativas, referentes aos seguintes aspectos: aprendizagem permanente do médico; auto-aprendizagem; avaliação do processo de aprendizagem; e aprendizagem cooperativa;

Dimensão ética: 16 itens, sendo 8 representativos de atitudes positivas e 8 de atitudes negativas, referentes a aspectos de comportamento ético enquanto acadêmico e em relação à ética médica codificada nacionalmente.

A versão preliminar do instrumento foi aplicada a oito estudantes com características pessoais de liderança, de bom desempenho acadêmico e que se dispuseram a colaborar com a pesquisa, bem como contribuir com sugestões relativas à apresentação da escala e explicações necessárias à formulação de item que expressasse o que se pretendeu mensurar nas respostas dos sujeitos pesquisados. O critério utilizado para a ordem dos itens que compõem a escala foi o sorteio. Com base nas sugestões dos estudantes foi aperfeiçoada a redação para melhor adequação dos itens e subseqüente aplicação na amostra, mantendo-se a ordem sorteada. 
A coleta de dados foi realizada em dois momentos: sala de aula e sala de tutorial. As salas tinham boa iluminação, equipamentos (mesas e cadeiras) e ventilação de ar adequados.

O tempo necessário para os pesquisados responderem o questionário foi em média de 25 minutos em cada reunião, conforme a previsão inicial.

A explicação sobre a base geral dos instrumentos utilizados (objetivo, forma e conteúdo) foi dada com a neutralidade requerida nas investigações de cunho científico, em linguagem simples e objetiva. É importante mencionar que foram solicitadas a adesão voluntária e a assinatura do termo de consentimento pós-informado, com o objetivo de garantir a privacidade do pesquisado.

Em seguida, a escala correspondente a cada item foi apresentada aos estudantes. Das cinco opções existentes, os pesquisados deveriam escolher a que melhor expressasse sua opinião sobre cada assertiva, isto segundo a intensidade de sua concordância ou discordância, conforme o seguinte esquema: 1 - Estou totalmente em desacordo; 2 - Discordo em parte; 3 - Estou em dúvida; 4 - Concordo em parte; 5 - Estou totalmente de acordo. Na oportunidade informou-se também que não havia respostas "certas" ou "erradas", uma vez que a pesquisa objetivava analisar tendências atitudinais.

Após o preenchimento, a escala de atitudes foi colocada em um envelope identificado, lacrado pelo próprio estudante e depois arquivado. Esse envelope foi entregue ao estudante no segundo momento da coleta de dados. Nessa oportunidade aplicou-se novamente a escala de atitude, seguindo a mesma metodologia. Ato seguinte, o estudante colocou os documentos gerados pelos dois momentos da coleta de dados em um envelope sem nenhuma identificação. Tal procedimento tinha o fito de garantir o sigilo e a proteção da identidade do respondente e, ao mesmo tempo, possibilitar a avaliação da "atitude" de maneira individualizada.

As categorias de declarações receberam valores de um a cinco, de acordo com o nível de concordância de cada item. Para a análise dos resultados, as respostas para os itens que expressavam atitudes positivas receberam valor de um a cinco para as cinco opções, conforme a intensidade da concordância expressa pelos sujeitos pesquisados. Do mesmo modo, as respostas aos itens que expressavam atitudes negativas receberam pontuação de um a cinco, em sentido inverso.

Assim, determinou-se a média de pontos obtida pelos estudantes em cada fase em relação ao conteúdo da escala. Com um total de 102 itens na escala construída, a média variou entre 102 e 510 pontos. Os pontos obtidos na escala foram considerados como variáveis quantitativas e utilizaram faixas de pontos associados a termos lingüísticos para a interpretação da atitude: negativa (102 a 305 pontos), conflitante (306 a 407 pontos) e positiva (408 a 510 pontos).

Para identificar a tendência atitudinal de cada sujeito da amostra foi obtida uma média geral dos escores (M) para a resposta de cada item da escala. Os resultados então expressos foram interpretados da seguinte maneira:

$-\mathrm{M}<3$ = atitude negativa

- M 3 a 4 = atitude conflitante

$-\mathrm{M}>4$ = atitude positiva

A análise da confiabilidade da escala foi realizada tomando-se por base o coeficiente de \&\#945 de Cronbach. Num primeiro momento aplicou-se a todos os itens da escala (escala atitude) e posteriormente apenas aos itens que compuseram as dimensões (subescala ou dimensões).

Para investigar a possibilidade de redução de itens na escala de atitude foi também analisada a confiabilidade pelos métodos das metades e pelo coeficiente de Guttmann e Flanagan.

Com a análise de agrupamento realizada por meio do método de K médias (cluster) investigou-se a existência de grupos de estudantes com atitudes "diferentes" entre si.

A análise fatorial também foi utilizada para investigação de evidências de atitudes diferenciadas em itens do conjunto dos itens da escala e das dimensões.

Os dados coletados foram estruturados com o auxílio dos softwares Epi Info 6.04 e Excel 7.0 (Microsoft). Para a análise estatística empregou-se o programa Statistica 5.0 (Statsoft).

O nível de significância da série estatística analisada foi da ordem de $5 \%$ ou 0,05 .

Por último, é importante mencionar que a pesquisa, desde a definição da escala de atitude utilizada e sua validação externa, a aplicação do instrumento de pesquisa e a análise dos resultados, se desenvolveu de dezembro de 2003 a dezembro de 2005 .

\section{RESULTADOS}

Os sujeitos da pesquisa foram 202 estudantes e deste total $52,85 \%$ eram mulheres e $47,15 \%$ homens. A média de idade foi de 21,45 anos (17 a 45 anos), não apresentando diferenças significativas na média de idade quanto à fase que o estudante estava cursando.

A clusterização (ou agrupamento) identificou dois conjuntos de estudantes que apresentaram atitudes semelhantes entre os sujeitos do mesmo grupo, porém diferentes entre os diversos grupos, mas esta diferença foi pequena. 
A análise fatorial demonstrou que o comportamento dos estudantes na aplicação da escala de atitude no início do semestre apresentou certa homogeneidade. Foram observados comportamentos diferenciados dos estudantes em poucos itens.

Os sumários estatísticos da escala de atitudes e das dimensões atitudinais propostas são apresentados nas Tabelas 1 e 2, respectivamente. A Tabela 3 apresenta a identificação e a correlação dos 51 itens que constituíram a metade A da escala de atitude. A Tabela 4 apresenta a distribuição dos estudantes em faixas de variação da média dos escores relativamente à aplicação da pesquisa no início e no final do semestre letivo. A média dos pontos obtidos pelos estudantes segundo a fase cursada é demonstrada na Tabela 5 .

TABELA 1

Consistência interna para a escala de atitude de estudantes de um curso de graduação em Medicina - Santa Catarina, 2006

\begin{tabular}{lcc}
\hline \multirow{2}{*}{ Sumário estatístico } & \multicolumn{2}{c}{ Escala de atitude } \\
\cline { 2 - 3 } & Início* $^{*}$ & Final* $^{*}$ \\
\hline Número de itens & 102,00 & 102,00 \\
Média (pontos) & 421,47 & 414,78 \\
Desvio padrão (pontos) & 26,08 & 31,68 \\
Mínimo (pontos) & 333,00 & 331,00 \\
Máximo (pontos) & 478,00 & 472,00 \\
$\alpha$ de Cronbach & 0,87 & 0,89 \\
Guttmann-Flanagan & 0,86 & 0,89 \\
Método das metades & 0,86 & 0,89 \\
\hline
\end{tabular}

* Relativo ao período de aplicação da escala no semestre letivo.

TABELA 2

Consistência interna para as dimensões da escala de atitude em estudantes de um curso de graduação em Medicina - Santa Catarina, 2006

\begin{tabular}{l|c|c|c|c|c}
\hline \multirow{2}{*}{ Dimensão } & \multirow{2}{*}{$\begin{array}{c}\text { Número } \\
\text { de Itens }\end{array}$} & \multirow{2}{*}{$\begin{array}{c}\alpha \text { de } \\
\text { Cronbach }\end{array}$} & \multicolumn{2}{|c|}{ Pontos } & \multirow{2}{*}{ n. } \\
\cline { 4 - 5 } & & & Média & DP & válidos \\
\hline Social & 24 & 0,69 & 103,72 & 7,89 & 193 \\
Ambiência & 16 & 0,57 & 64,07 & 6,01 & 193 \\
Crença & 16 & 0,53 & 63,29 & 6,42 & 196 \\
Conhecimento & 30 & 0,66 & 127,39 & 9,45 & 194 \\
Ética & 16 & 0,49 & 63,92 & 6,14 & 195 \\
\hline
\end{tabular}

Nota 1 - DP = desvio padrão ; Nota 2 - $\mathrm{n}$ = número de respondentes
TABELA 3

Escala de atitudes: distribuição dos estudantes em faixas de variação da média dos escores nos diferentes itens referentes à aplicação no início e fim do semestre letivo. Curso de graduação em Medicina - Santa Catarina, 2006

\begin{tabular}{l|c|c|c}
\hline \multirow{4}{*}{ Atitude } & Média do & \multicolumn{2}{|c}{ Amostra } \\
\cline { 3 - 4 } & Escore* $^{*}$ & $\mathbf{n}$ & $\%$ \\
\hline \multirow{3}{*}{$\begin{array}{l}\text { Conflitante } \\
\text { Início* }\end{array}$} & 51 & 25,25 \\
Positiva & $3,0-3,99$ & 151 & 74,75 \\
& $4-5,0$ & 202 & 100,00 \\
& Total & & \\
Conflitante & & & \\
Positiva & $3,0-3,99$ & 124 & 38,61 \\
& $4-5,0$ & 202 & 61,39 \\
& Total & \multicolumn{2}{c}{100,00} \\
\hline
\end{tabular}

* Relativo ao período da aplicação da escala no semestre letivo.

TABELA 4

Média dos pontos da escala de atitudes apresentados pelos estudantes de oito fases em um curso de graduação em Medicina - Santa Catarina, 2006

\begin{tabular}{l|c|c}
\hline \multirow{2}{*}{ Fase } & \multicolumn{2}{|c}{ Escala de Atitude $(\mathbf{n}=\mathbf{1 7 3})$} \\
\cline { 2 - 3 } & $\mathbf{M}$ & $\mathbf{D P}$ \\
\hline Primeira & 433,79 & 27,55 \\
Segunda & 414,38 & 21,15 \\
Terceira & 430,35 & 22,02 \\
Quarta & 416,41 & 35,55 \\
Quinta & 420,47 & 19,20 \\
Sexta & 430,80 & 25,91 \\
Sétima & $405,96^{*}$ & 30,81 \\
Oitava & 420,74 & 29,47 \\
Amostra & 421,47 & 28,08 \\
\hline
\end{tabular}

$\mathrm{n}$ = número de respondentes; $\mathrm{DP}=$ desvio padrão; $\mathrm{M}=$ média dos pontos; ${ }^{*} \mathrm{p} \leq 0,01$

Classificação da escala de atitude conforme a pontuação obtida: 306 a 407 conflito; 408 a 510: positiva.

TABELA 5

Média dos pontos da escala de atitudes, apresentados pelos estudantes de oito fases em um Curso de Graduação em Medicina - Santa Catarina, 2006.

\begin{tabular}{l|c|c}
\hline \multirow{2}{*}{ Fase } & \multicolumn{2}{c}{$\begin{array}{c}\text { Escala de Atitude } \\
\text { n=173 }\end{array}$} \\
\cline { 2 - 3 } & M.. & DP \\
\hline Primeira & 433,79 & 27,55 \\
Segunda & 414,38 & 21,15 \\
Terceira & 430,35 & 22,02 \\
Quarta & 416,41 & 35,55 \\
Quinta & 420,47 & 19,20 \\
Sexta & 430,80 & 25,91 \\
Sétima & $405,96^{*}$ & 30,81 \\
Oitava & 420,74 & 29,47 \\
Amostra & 421,47 & 28,08 \\
\hline
\end{tabular}

$\mathrm{n}=$ número de respondentes $\mathrm{DP}=$ desvio padrão

$\mathrm{M}=$ média dos pontos ${ }^{*} \mathrm{p}<0,01$

Classificação da escala de atitude conforme a pontuação

obtida: 306 a 407: conflito; 408 a 510: positiva 


\section{DISCUSSÃO}

Diferentes escalas foram construídas para a mensuração de atitudes de estudantes e profissionais da saúde. Algumas abordavam comportamentos favoráveis a segmentos sociais ou a práticas isoladas, ao passo que outras abordavam aspectos gerais do estudante/profissional médico.

Considerando a filosofia geral da profissão médica, é importante destacar as escalas desenvolvidas por Parlow e Rothmann $^{8}$, que abordam os aspectos sociais da medicina (Attitudes Toward Social Issues in Medicine, ATSIM), a de atitude em relação à habilidade de comunicação de Rees, Sheard e Davies ${ }^{13}$ (Communications Skills Attitude Scale, CSAS) e a desenvolvida por Colares e outros ${ }^{14}$, que aborda aspectos relacionados à prática geral médica.

Esta pesquisa teve como foco o desenvolvimento e validação de uma escala para mensuração de atitudes de estudantes de Medicina, para a observação e percepção do desenvolvimento das mesmas durante o curso de graduação.

Ressalte-se que bons instrumentos para avaliação de atitudes devem ser capazes de apresentar uma medida quantitativa da posição relativa do indivíduo, sem o que não é possível comparar as diferenças ou identificar mudanças de atitudes ${ }^{2,4,6,12}$.

A opção por construir uma escala do tipo Likert decorreu do fato de tal instrumento de pesquisa ser considerado fidedigno $^{2,3}$, com validade igual ou maior do que as demais ${ }^{2,4}$, permitindo uma informação mais precisa sobre o indivíduo com relação à questão apresentada por um item ${ }^{4}$.

Outro aspecto a destacar diz respeito ao fato de a referida escala ser considerada uma construção mais simples ${ }^{2,4-6}$, além de ter sido usada amplamente em estudos de atitude em geral e em pesquisa para mensurações de aspectos atitudinais de estudantes de Medicina.

Contudo, quaisquer generalizações devem levar em conta algumas restrições próprias da escala do tipo Likert, pois, apesar de permitir ordenar indivíduos por meio de sua favorabilidade a determinado aspecto atitudinal, essa escala não apresenta uma base sobre quanto um indivíduo é mais favorável que outro ${ }^{2-6,18}$.

Também há que considerar o fato de freqüentemente o escore total de um indivíduo não apresentar um significado muito claro, pois diferentes padrões de respostas podem conduzir ao mesmo escore ${ }^{2,4}$. O próprio sujeito da pesquisa pode determinar um fator de erro quando apresenta o desejo de uma imagem socialmente aceitável ou uma tendência a concordar (ou discordar) com afirmações independentes do seu conteúdo $^{1-6,18}$. Para restringir esses possíveis fatores de erro na construção da escala, precisou-se considerar os critérios de conteúdo e de construto.
Os dados indicaram que a escala proposta apresenta validade e fidedignidade satisfatórias. O coeficiente de \&\#945 de Cronbach encontrado no período de aplicação da pesquisa (no início e no final do semestre $(0,87$ e 0,89$)$ pode ser considerado um resultado satisfatório.

O índice obtido no teste de correlação das metades (0,86 e $0,89)$ pode ser considerado razoável e demonstra fidedignidade aceitável para cada metade da escala de atitude. O mesmo fato foi demonstrado pelo índice de Guttmann-Flanagam (0,86 e 0,89 ). Esses resultados indicam a possibilidade de dividir a escala de atitudes em duas metades, cada uma com 51 itens.

A aceitação da escala pelos estudantes ficou demonstrada no alto índice de resposta aos itens pesquisados.

Streit ${ }^{9}$ e Woloschuk e outros ${ }^{17}$, em estudos de coorte para mensuração de aspectos atitudinais em estudantes de Medicina, consideraram que escores atitudinais altamente positivos encontrados em suas pesquisas poderiam ser decorrentes da existência das mesmas atitudes já por ocasião do ingresso no curso.

O estudo evidenciou maior percentual de estudantes com tendência atitudinal positiva. Foram observados resultados semelhantes no início e no final do semestre letivo. Tal resultado, no entanto, era esperado, já que o período de um semestre letivo é considerado curto para a ocorrência de mudanças atitudinais. As atitudes, apesar de serem mutáveis e de a ambiência da aprendizagem apresentar fatores que concorrem para a mudança, não são fáceis de mudar ou substituir.

A tendência atitudinal positiva ficou demonstrada quando foi observada a média dos pontos obtida na escala de atitudes no início do semestre letivo. A provável homogeneidade do comportamento dos sujeitos pesquisados pode ser verificada no desvio padrão da média da pontuação encontrado na análise da amostra e mesmo na análise realizada por fase

\section{CONCLUSÃO}

O trabalho desenvolvido resultou na definição de uma escala de atitude com validade e confiabilidade adequadas para a medida de atitudes em estudantes de graduação em Medicina, podendo ser dividida em duas metades para utilização em outras pesquisas.

Entre as diversas possibilidades do uso de uma escala de atitude, uma seria o monitoramento das atitudes de estudantes de Medicina durante a graduação, o que permitiria investigar mudanças atitudinais destes estudantes, assim como indicadores de impacto curricular e vivências acadêmicas.

As escolas médicas precisam conhecer melhor o desenvolvimento de atitudes e possíveis mudanças, de modo que possam atender às demandas sociais e promover as necessárias mudanças na profissão médica. 


\section{LIMITAÇÕES}

Esta pesquisa foi realizada em apenas uma instituição, portanto limitando possíveis generalizações.

\section{REFERÊNCIAS}

1. Miller GE.(Org.) Ensino e aprendizagem nas escolas médicas. Tradução de Maria Helena Caldas de Oliveira. São Paulo: Nacional; 1967.

2. Summers GF; Bohrnstedt GW. Medicion de actitudes. México: Trillas; 1976.

3. Lambert W W, Lambert, W E. Psicologia social. Rio de Janeiro: Zahar; 1981.

4. Selltiz C. Métodos de pesquisa nas relações sociais. São Paulo: EPU; 1987. v.2.

5. Pasquali L. Psicometria: teoria e aplicações. Brasília: UnB; 1997.

6. Cronbach LJ. Fundamentos da Testagem Psicológica. Do original Essentials of Psychological Tests. Tradução de Carlos Alberto Silveira Neto e Maria Adriana Veríssimo Veronese. Porto Alegre: Artes Médicas; 1996.

7. Rodrigues A. Psicologia Social para principiantes. Petrópolis: Vozes; 2002.

8. Parlow J, Rothman AI. ATSIM: a scale to measure attitudes towards psychosocial in health care. J. Med Educ. 1974;49: 385-6.

9. Streit U. Attitudes towards psycosocial factors in medicine: an apraisal of the ATSIM sacale. Med Educ. 1980;14:259-66.

10. Burra P, Kalin R, Leichener S, Waldron J, Handforth JR, Jarret FJ et al. The ATP-30: a scale for measuring medical students' attitudes to psiquiatry. Med Educ. 1982;16:31-8.

11. Parsell G, Bligh, J. The development of a questionnaire to assess the readiness of health care students for interprofessional learning (RIPLS). Med Educ. 1999;33(2):95-100.

12. Rolfe IE, Pearson SA, Cleary EG, Gannon C. Attitudes towards community medical: a comparison of students from traditional and community-oriented medical schools. Med Educ. 1999;33:606-11.

13. Rees C, Sheard C, Davies S. The development of a scale to measure medical students' attitudes towards communication skills learning: the Communication Skills Attitude Scale (CSAS). Med Educ. 2002;36(2):141-7.

14. Colares MFA, Troncon LEA, Figueiredo JFC, Cianflone $\mathrm{ARC}$, Rodrigues MLV, Piccinato CE et al. Construção de um instrumento para avaliação das atitudes de estudantes de medicina frente a aspectos relevantes da prática médica. Rev Bras de Educ Méd. 2002;26(3):194-203.

15. Masson N, Lester H. The attitudes of medical students towards homeless people: does medical school make a difference? Med Educ. 2003;37(10):869-72.

16. Hren D, Lukic IK, Marusic A, Vodopivec I, Vujaklija A, Hrabak $M$ et al. Teaching research methodology in medical schools: students' attitudes towards and knowledge about science. Med Educ. 2004;38(1):81-6.

17. Woloschuk W, Harasym PH, Temple W. Attitude change during medical school: a cohort study. Med Educ 2004;38(5):522-34.

18. Pasquali, L. Princípios de elaboração de escalas psicológicas / Principles of elaboration of psychological scales. São Paulo: Rev. Psiquiatr. Clin. 1998;25(5):206-13.

\section{CONFLITOS DE INTERESSE}

Declarou não haver.

\section{ENDEREÇO PARA CORRESPONDÊNCIA}

Silvana Maria de Miranda

Rua José Bonifácio, 185 - apt. 603 - Centro

CEP. 88 802-140 - Criciúma - SC

E-mail: silvanamiranda@terra.com.br 